\title{
ERRATUM
}

Georgios Tsounis $\cdot$ Sergio Rossi $\cdot$ Maria Aranguren

Josep-Maria Gili • Wolf Arntz

\section{Effects of spatial variability and colony size on the reproductive output and gonadal development cycle of the Mediterranean red coral (Corallium rubrum L.)}

Published online: 26 January 2006

(C) Springer-Verlag 2006

\section{Marine Biology (2006) 148:513-527}

Unfortunately Table 3 contained errors. The correct

Table 3 is shown below:

Table 3 Size/age at first reproduction in various gorgonians

\begin{tabular}{|c|c|c|c|c|}
\hline \multirow[t]{2}{*}{ Species } & \multirow[t]{2}{*}{ Location } & \multicolumn{2}{|c|}{ First reproduction } & \multirow[t]{2}{*}{ Source } \\
\hline & & Age (years) & Height $(\mathrm{cm})$ & \\
\hline Antipathes dichotoma & Hawaii & $10-12.5$ & $64-80$ & Grigg 1977 \\
\hline Briareum asbestinum (branches) & Caribbean & $2-3$ & $10-20$ & Brazeau and Lasker 1990 \\
\hline Corallium rubrum & Mediterranean & - & 2 & This study \\
\hline Corallium rubrum & Mediterranean & 2 & 2 & Santangelo et al. 2003b; Bramanti 2003 \\
\hline Monastrea annularis & Caribbean & 4 & - & Szmant-Froehlich 1985 \\
\hline Muricea californica & California & 5 & 12 & Grigg 1974, 1977 \\
\hline Muricea fructicosa & California & 10 & 20 & Grigg 1974, 1977 \\
\hline Paramuricea clavata & Mediterranean & & 15 & Coma et al. $1995 \mathrm{a}$ \\
\hline Parerythropodium fulvum fulvum & Red Sea & $3-4$ & - & Benayahu and Loya 1984 \\
\hline Plexaura $A$ & Caribbean & 5 & 20 & Brazaeu and Lasker 1989 \\
\hline Plexaura flexuosa & Caribbean & - & $20-30$ & Beiring and Lasker 2000 \\
\hline Plexaura homomalla & Caribbean & - & 20 & Brazaeu and Lasker 1989 \\
\hline Sarcophytum glaucum & Red Sea & $7-10$ & - & Brnayahu and Loya 1984 \\
\hline Stylophora pistillata & Red Sea & 2 & - & Rinkevich and Loya 1979 \\
\hline Xenia macrospiculata & Red Sea & 2 & 1 & Benayahu and Loya 1984 \\
\hline
\end{tabular}

The online version of the original article can be found at http:// dx.doi.org/10.1007/s00227-005-0100-8

G. Tsounis · W. Arntz

Alfred Wegener Institute for Polar and Marine Research,

Columbusstr., 27568 Bremerhaven, Germany

S. Rossi $(\bowtie) \cdot$ M. Aranguren $\cdot$ J.-M. Gili

Institute de Ciéncies del Mar (CSIC),

Passeig Marítim de la Barceloneta 37-49,

08003 Barcelona, Spain

E-mail: srossi@icm.csic.es 